internationally. However, morbidity data are now considered more relevant to current public health concerns, though inherently less accessible. We show how linkage of routine health data can supplement and complement mortality data to produce information that is pertinent to both research and policy making. We use diabetes, a worldwide chronic disease, driven by trends in obesity and ageing populations, as an exemplar. There are an estimated 285 million adults aged 20-79 living with diabetes worldwide yet there is a paucity of successful surveillance tools to measure the burden of this disease. Between March 2009 and March 2010 3.4\% of the population cashed prescriptions for anti-diabetic medication $(n=63853)$ with multilevel analysis showing an increased association with older age, males and increased deprivation. This study shows how the prevalence of diabetes in one country (Northern Ireland-c1.8 million) has been estimated from prescription data relating to treated diabetes. These estimates will be strengthened with linkages to data from laboratories measuring diabetic control and data from primary care used to inform the diabetic retinopathy screening services.

\section{P1-25 POTENTIAL BIAS IN HIV ESTIMATES USING RDS SAMPLING}

doi:10.1136/jech.2011.142976c.19

M Guimaraes, * C Machado. Federal University of Minas Gerais, Belo Horizonte, Minas Gerais, Brazil

Introduction Respondent-driven sampling (RDS) is a process used to collect data from hard-to-reach populations, such as men who have sex with men (MSM). This is a process of accessing a hidden population of interest via following links in the network of acquaintances belonging to the population, and it can be a useful epidemiological tool for estimating HIV prevalence in high risk populations. However, a typical sample arising from an RDS process on a network contains a certain degree of homophily, and in case the quantity surveyed is a given characteristic, this can generate biased results.

Methods A cross-sectional study was conducted in Belo Horizonte, Brazil, in order to estimate the HIV prevalence among MSM in 2009. Recruitment was carried out using RDS sampling. We assessed whether HIV status of the recruiter was associated with HIV status of the recruitee. Statistical analysis was carried out by McNemaŕs $\chi^{2}$.

Results We analysed 221 recruiter-recruitee pairs among the 274 MSM included in the study. The prevalence of HIV infection was $10.8 \%$ (95\% CI 4.5 to 17.8 ). The probability of recruiting an HIV positive individual was $80 \%$ when the recruiter was also HIV positive and $8.3 \%$ when the recruiter was HIV negative, indicating a dependency on HV status during the recruitment process (McNemar=13.5, $\mathrm{p}<0.001)$.

Conclusion Although RDS sampling may be suitable for ascertaining HIV prevalence among hidden populations, these estimates are likely to be biased and results should be carefully assessed and proper adjustments carried out.

\section{P1-26 THE ALL IRELAND TRAVELLER HEALTH STUDY OF TEN THOUSAND FAMILIES PROVIDES A HUMAN DEVELOPMENT INDEX FOR IRISH TRAVELLERS}

doi:10.1136/jech.2011.142976c.20

N A Hamid, ${ }^{*}$ C Kelleher, L Daly, B Quirke, P Fitzpatrick, For the All Ireland Traveller Health Study Team. University College Dublin, Belfield, Dublin 4, Ireland

Introduction Irish Travellers, a nomadic minority group in Ireland, experience multi-faceted social deprivation. The All Ireland Traveller
Health Study (AITHS) 2007-2011 of an estimated 10000 families in both Irish jurisdictions shows little improvement in most measured indicators since the last systematic assessment in 1987. The Human Development Index (HDI), measures long term basic dimensions including life expectancy, access to knowledge and an adequate standard of living. In 2010, using the improved HDI calculation, Republic of Ireland (ROI) was placed fifth (HDI=0.895) in the world league (2005-2009: 0.894). The aim of this analysis is to calculate the HDI of Irish Travellers for 2008.

Methods The AITHS census, a comprehensive 45 min census interview employing a novel audio-visual computer-assisted instrument, was conducted with Traveller families by 180 trained peer researcher teams over a 6 week period commencing on $14^{\text {th }}$ October 2008. A retrospective mortality study over past 1 year was also conducted. Data from both were used to construct the HDI for Irish Travellers.

Results The family response rate was 79\%. Life expectancy at birth was estimated at 61.7 years for men, 70.1 for women. Mean years of schooling was 8.1 (SD 4) and 94\% of Irish Travellers were below the poverty line. Calculated HDI for Irish Travellers in 2008 is 0.713 , 0.181 below ROI's HDI.

Conclusion There is a $20.2 \%$ difference in HDI for Travellers compared to the general population. This is wider than seen for other indigenous minority populations worldwide and has important cross-sectoral policy implications. (250 words).

\section{P1-27 EFFECTIVENESS OF TRAINING IN MOTIVATIONAL INTERVIEWING IN OUITTING OR REDUCING SMOKING INTENSITY IN PREGNANCY AND POST-PARTUM}

doi:10.1136/Jech.2011.142976c.21

${ }^{2} \mathrm{C}$ Hayes, ${ }^{*}{ }^{3} \mathrm{C}$ Collins, ${ }^{3} \mathrm{H}$ O'Carroll, ${ }^{3} \mathrm{E}$ Wyse, ${ }^{2} \mathrm{M}$ Gunning, ${ }^{2} \mathrm{~A}$ Rhatigan, ${ }^{3} \mathrm{M}$ Geary, ${ }^{1} \mathrm{C}$ Kelliher. ${ }^{1}$ University College Dublin, Dublin, Ireland; ${ }^{2}$ Health Service Executive, Dublin, Ireland; ${ }^{3}$ Rotunda Hospital, Dublin, Ireland

Introduction This study aimed to determine whether an integrated approach, involving training of professional staff in maternity and child health services to deliver sustained brief intervention using Motivational Interviewing (MI) techniques in a standardised way, was sufficient to affect change in smoking status or intensity in lowincome pregnant and post-partum women.

Methods 500 consecutive smokers were recruited at the first antenatal visit to receive usual care (controls). Post staff training, 500 more women were recruited (intervention group). Data were recorded at 28-32 weeks gestation and 3-4 months post-partum. The primary outcome measure was self-reported smoking cessation verified by urinary cotinine. Changes in smoking intensity were also measured.

Results There was no difference in the proportion of those who stopped smoking at $28-32$ weeks pregnancy $(8.8 \%$ vs $9.3 \%) \chi^{2}=5.9$, d.f. $=3$, $p=0.12$, or $3-4$ months post-partum $7.6 \%$ vs $7.2 \%\left(\chi^{2}=4.46\right.$, d.f. $=3, p=0.21)$. In late pregnancy cases that had stopped at baseline were more likely to stay stopped ( $26 \%$ vs $42 \%$ ). More cases who were light ( $<5$ cigs per day) or medium intensity (5-10 per day) smokers at baseline were non-smoking in late pregnancy (light, $21.3 \%$ vs $15.8 \%$, medium $6.3 \%$ vs $3.1 \%$ ). At $3-4$ months postpartum more cases smoking 5-15 cigarettes per day moved to a lower intensity $(83.7 \%$ vs $52.1 \%$ ) and fewer shifted to a higher smoking category.

Conclusion Brief intervention of the intensity offered was ineffective in affecting quit rates in late pregnancy and 3-4 months postpartum. Subtle positive changes around smoking intensity were observed. 